\title{
Efeito de diferentes protocolos de superovulação sobre a concentração plasmática de progesterona e de metabólitos lipídicos de vacas Nelore
}

\author{
[Effect of different superovulation protocols on plasma progesterone concentration and \\ lypidic metabolites in Nelore cows] \\ A.F. Ramos $^{1,4}$, E.F. Neves ${ }^{1}$, V.S. Marques ${ }^{1}$, F.P.C. Lima ${ }^{1}$, D.L. Drumond ${ }^{2}$ \\ A.P. Marques $J r^{3 *}$ \\ ${ }^{1}$ Aluno de pós-graduação - EV-UFMG \\ ${ }^{2}$ Médico veterinário autônomo \\ ${ }^{3}$ Escola de Veterinária - UFMG \\ Caixa Postal 567 \\ 30123-970 - Belo Horizonte, MG \\ ${ }^{4}$ Bolsista da CAPES
}

\begin{abstract}
RESUMO
Avaliaram-se as concentrações plasmáticas de triglicérides, colesterol, aspartato transaminase (AST) e progesterona ( $\mathrm{P} 4)$ em vacas Nelore não lactantes com elevado escore corporal, superovuladas com diferentes protocolos. Foram utilizados três grupos de animais, G1 $(n=11)$, G2 (n=8) e G3 (n=5), superovulados com 500UI de FSH, 200mg e 180mg de FSH (hormônio folículo estimulante), respectivamente, em doses decrescentes, duas vezes ao dia, durante quatro dias. As amostras de sangue foram coletadas antes da superovulação (A), no terceiro dia da superovulação (B), no momento da inseminação artificial (C) e na coleta dos embriões (D). As concentrações de triglicérides, AST e colesterol foram verificados por espectrofotometria, e a de progesterona (P4) por radioimunoensaio. Não houve alteração $(\mathrm{P}>0,05)$ na concentração de triglicérides, AST e colesterol entre as amostras. Não houve efeito $(\mathrm{P}>0,05)$ do protocolo de superovulação sobre a concentração de triglicérides, AST e P4 nas diferentes amostras. O G2 apresentou menor concentração de colesterol $(\mathrm{P}<0,05)$ nas amostras $\mathrm{A}$ e $\mathrm{B}$, possivelmente em razão da grande instabilidade dessa variável.
\end{abstract}

Palavras-chave: vaca, Nelore, aspartato transaminase, colesterol, progesterona, superovulação, triglicéride

\begin{abstract}
The purpose of this research was to evaluate the concentration of tryglicerides, cholesterol, aspartate transaminase (AST) and progesterone (P4) in embryo donor Nelore cows superovulated with different protocols. Twenty four donors were randomly distributed in three groups: group 1 ( $n=11)$, donors superovulated with 500UI of FSH and group $2(n=8)$ and group $3(n=5)$ respectively with $200 \mathrm{mg}$ and $180 \mathrm{mg}$ of FSH, in decreasing doses, twice a day, during four consecutive days. Blood samples were collected before superovulation $(A)$, in the third day of superovulation $(B)$, at the artificial insemination time $(C)$ and at the embryo collection time (D). The concentrations of tryglicerides, aspartate transaminase (AST) and cholesterol were measured by spectrophotometry and progesterone (P4) by radioimmunoassay. There was no alteration $(P<0.05)$ in the concentration of tryglicerides, AST and cholesterol among the samples. There was no effect $(P>0.05)$ of the superovulation protocol on the concentration of tryglicerides, AST and P4 in the samples. In the samples $A$ and $B$ of group 2 the concentration of cholesterol was lower $(P<0.05)$ than in groups 1 and 3 , probably due to the instability of the parameter.
\end{abstract}

Keywords: cow, Nelore, aspartate transaminase, cholesterol, progesterone, superovulation, trygliceride

Recebido em 24 de setembro de 2006

Aceito em 21 de dezembro de 2006

*Autor para correspondência (corresponding author)

e-mail: ampinho@ufmg.br 


\section{INTRODUÇÃO}

As biotecnias reprodutivas têm sido utilizadas para acelerar a multiplicação de genes superiores, e o sucesso dos programas de transferência de embriões depende, em grande parte, da resposta à superovulação, seu fator limitante (Neves et al., 2005).

Estudos sobre o perfil metabólico e bioquímico do sangue de vacas tiveram início na década de 70 , e seu uso possibilita entender as relações entre os componentes sangüíneos e eventos fisiológicos, bem como identificar rebanhos com problemas metabólicos que afetam direta ou indiretamente a fertilidade e a produtividade dos mesmos.

Constituintes bioquímicos do sangue, como colesterol, triglicérides e progesterona, podem interferir na eficiência reprodutiva do animal. O colesterol, um dos principais componentes estruturais da membrana plasmática das células dos mamíferos, é importante para o crescimento e a sobrevivência celular, além de ser o precursor dos hormônios esteróides. Os triglicérides, maiores componentes lipídicos do tecido adiposo, são constituídos por três ácidos graxos de cadeia longa esterificados a uma molécula de glicerol, com capacidade de aumentar a concentração plasmática de colesterol (Wathes et al., 1998). A progesterona, secretada principalmente pelo corpo lúteo e pela placenta, tem como principais funções inibir a secreção de gonadotropinas na fase luteal do ciclo estral e preparar o trato reprodutivo para a implantação embrionária e a manutenção da gestação, pelo estímulo secretório às glândulas uterinas e inibição da motilidade do miométrio (Hadley, 1996).

O objetivo deste experimento foi caracterizar o efeito de diferentes protocolos de superovulação sobre parâmetros bioquímicos do sangue em vacas Nelore com elevado escore corporal.

\section{MATERIAL E MÉTODOS}

$\mathrm{O}$ experimento consistiu na avaliação de parâmetros bioquímicos do sangue, incluindo triglicérides, aspartato transaminase, colesterol e progesterona, em doadoras de embrião da raça Nelore, não-lactantes, do início da superovulação até a coleta dos embriões. O experimento foi realizado nos meses de junho e julho, caracterizados por um período de seca.

Foram utilizadas 24 doadoras, com escore da condição corporal de 7 a 9 numa escala de 1 a 9 pontos, segundo Dias (1991). O grupo $1(\mathrm{n}=11)$ foi superovulado com 500UI de $\mathrm{FSH}^{1}$, e os grupos $2(n=8)$ e $3(n=5)$ com 200 e $180 \mathrm{mg}$ de $\mathrm{FSH}^{2}$, respectivamente. Os protocolos de superovulação foram feitos com administração de doses decrescentes de FSH, duas vezes ao dia, do dia 9 (dia 0 = dia do estro) até o dia 12 do ciclo estral, com aplicação de análogo de prostaglandina $\mathrm{F} 2 \alpha^{3}$ nos dias 11 e 12 (Tab. 1).

As vacas foram mantidos em pastagem de Brachiaria brizanta, sem suplementação, recebendo água e sal mineral à vontade, e foram conduzidas ao curral dois dias antes do início do programa de superovulação, para avaliação e seleção daquelas consideradas aptas a serem superovuladas. $\mathrm{O}$ procedimento de seleção das doadoras, para entrar no programa de superovulação e coleta de embriões, foi realizado empiricamente por palpação transretal. Os animais que apresentavam corpo lúteo no momento da seleção (dia 7 do ciclo estral) foram submetidos à superovulação com início no dia nove do ciclo e subseqüente coleta dos embriões.

As amostras de sangue foram coletadas por venopunção jugular, antes do início da superovulação, para mensuração basal dos parâmetros (dia 9), no início do terceiro dia de superovulação (dia 11), por ocasião da primeira inseminação artificial (dia 13) e da coleta de embriões (dia 20). Para a coleta, foram utilizadas agulhas hipodérmicas ${ }^{4} 21 \mathrm{~g}$, tubos de $6 \mathrm{ml}$ com vácuo, contendo EDTA fluoretado e tubos de $8 \mathrm{ml} \mathrm{com} \mathrm{gel} \mathrm{separador,} \mathrm{ambos} \mathrm{comerciais}{ }^{5}$. Após a coleta, o sangue foi centrifugado ${ }^{6}$ a $4000 \mathrm{~g}$, durante cinco minutos. O plasma e o soro, acondicionados em tubos eppendorf de $1,5 \mathrm{ml} \mathrm{e}$ com três alíquotas de cada, foram estocados e congelados a $-20^{\circ} \mathrm{C}$ até análise de seus parâmetros metabólicos e hormonais.

\footnotetext{
${ }^{1}$ Pluset $\circledast$ - Laboratório Calier do Brasil

${ }^{2}$ Foltropin-V® - Laboratório Vetrepharm Inc./Tecnopec

${ }^{3}$ Cloprostenol sódico - Ciosin $®$ - Laboratório Coopers do Brasil -

${ }^{4}$ Precision Glide ${ }^{\circledR}$

${ }^{5}$ Vacuntainer $®$ - Becton Dickinson Ind. Cirúrgica Ltda

${ }^{6}$ Fanem ${ }^{\circledR}$ - Centréfuga Excelsa Baby - Mod.206
} 
Efeito de diferentes protocolos de superovulação...

Tabela 1. Protocolos de superovulação utilizados em diferentes grupos de vacas Nelore, doadoras de embrião, com elevado escore corporal (dia $0=$ dia do estro)

\begin{tabular}{|c|c|c|c|c|}
\hline \multirow{2}{*}{ Grupo } & \multicolumn{4}{|c|}{ Dias do ciclo estral } \\
\hline & 9 & 10 & 11 & 12 \\
\hline \multirow[t]{2}{*}{$\begin{array}{c}\text { 500UI FSH } \\
\quad(n=11)\end{array}$} & $\begin{array}{c}\text { 100UI FSH } \\
\text { manhã e tarde }\end{array}$ & $\begin{array}{c}\text { 75UI FSH } \\
\text { manhã e tarde }\end{array}$ & $\begin{array}{c}\text { 50UI FSH } \\
\text { manhã e tarde }\end{array}$ & $\begin{array}{c}\text { 25UI FSH } \\
\text { manhã e tarde }\end{array}$ \\
\hline & & & $\begin{array}{c}0,5 \mathrm{mg} \text { PGF } 2 \alpha \\
\text { tarde }\end{array}$ & $\underset{\text { tarde }}{0,5 \mathrm{mg} \text { PGF } 2 \alpha}$ \\
\hline \multirow[t]{2}{*}{$\begin{array}{c}200 \mathrm{mg} \mathrm{FSH}{ }^{2} \\
\quad(\mathrm{n}=8)\end{array}$} & $\begin{array}{c}\text { 40mg FSH } \\
\text { manhã e tarde }\end{array}$ & $\begin{array}{c}\text { 30mg FSH } \\
\text { manhã e tarde }\end{array}$ & $\begin{array}{c}\text { 20mg FSH } \\
\text { manhã e tarde }\end{array}$ & $\begin{array}{c}\text { 10mg FSH } \\
\text { manhã e tarde }\end{array}$ \\
\hline & & & $\begin{array}{c}0,5 \mathrm{mg} \text { PGF } 2 \alpha \\
\text { tarde }\end{array}$ & $\underset{\text { tarde }}{0,5 \mathrm{mg} \text { PGF } 2 \alpha}$ \\
\hline \multirow[t]{2}{*}{$\begin{array}{c}180 \mathrm{mg} \mathrm{FSH}{ }^{2} \\
(\mathrm{n}=5)\end{array}$} & $\begin{array}{c}\text { 36mg FSH } \\
\text { manhã e tarde }\end{array}$ & $\begin{array}{l}\text { 27mg FSH } \\
\text { manhã e tarde }\end{array}$ & $\begin{array}{c}18 \mathrm{mg} \text { FSH } \\
\text { manhã e tarde }\end{array}$ & $\begin{array}{c}\text { 9mg FSH } \\
\text { manhã e tarde }\end{array}$ \\
\hline & & & $\begin{array}{c}0,5 \mathrm{mg} \text { PGF } 2 \alpha \\
\text { tarde }\end{array}$ & $\begin{array}{c}0,5 \mathrm{mg} \text { PGF } 2 \alpha \\
\text { tarde }\end{array}$ \\
\hline
\end{tabular}

${ }^{1}$ Pluset ${ }^{\circledR}$ - Laboratório Calier; ${ }^{2}$ Foltropin-V® - Laboratório Vetrepharm

Os parâmetros bioquímicos, consistindo de triglicérides, aspartato transaminase e colesterol, foram determinados com o uso de kits comerciais $^{7}$ segundo os protocolos recomendados pelo fabricante. Todas as leituras colorimétricas foram feitas em aparelho de espectrofotometria $^{8}$, com comprimento de onda, para cada um dos parâmetros, sugerido pelo fabricante do kit comercial utilizado. Nos testes enzimáticos, para a estabilização da temperatura do complexo reagente-amostras, foi utilizado um banho-maria ${ }^{9}$. A análise de progesterona foi realizada através de radioimunoensaio utilizando kits comerciais ${ }^{10}$.

A metodologia estatística utilizada foi análise de variância e teste de Duncan, com auxílio do programa estatístico SAEG (Sistema..., 1995). Os resultados referentes à concentração de progesterona não apresentaram distribuição normal e sofreram transformação logarítmica.

\section{RESULTADOS E DISCUSSÃO}

O valor médio para a concentração sérica de colesterol foi de $137,80 \pm 52,06 \mathrm{mg} / \mathrm{dl}$, superior ao considerado fisiologicamente normal para vacas mestiças superovuladas (Borges et al., 2001) e vacas Nelore e Gir (Ruas, 1998; Borges et al., 2001). A concentração média de colesterol não variou do início da superovulação até a coleta

\footnotetext{
${ }^{7}$ Bioclin, Química Básica Ltda (Quibasa)

${ }^{8}$ Celm ${ }^{\circledR}$, R. Mod. E-225 D.

${ }^{9}$ Fanem ${ }^{\circledR}$ - Banho Maria Famen Mod. 100

${ }^{10}$ Bet laboratories
}

dos embriões, seja para os diferentes grupos como para a totalidade dos animais (Tab. 2). Os resultados sinalizam que, para este parâmetro, parece não haver efeito da superovulação sobre o metabolismo do animal. No entanto, o grupo tratado com $200 \mathrm{mg}$ de FSH apresentou menor concentração de colesterol $(\mathrm{P}<0,05)$ antes da superovulação e por ocasião da inseminação artificial em relação aos grupos tratados com 500UI de FSH e 180mg de FSH, possivelmente devido à instabilidade da variável estudada.

Guédon et al. (1999) sugeriram que a alta concentração de colesterol sangüíneo pode estar associada à melhor atividade ovariana em vacas no pós-parto, condição que não se aplica a este experimento, já que nenhum animal apresentavase nessa condição fisiológica. Não é possível definir se a concentração de colesterol alta, encontrada neste experimento, poderia estar favorecendo a esteroidogênese e melhorando a atividade ovariana ou se ela atingiu valores acima do tolerável, passando a ser prejudicial à atividade reprodutiva. O colesterol, através das lipoproteínas HDL e LDL, é a principal fonte para a esteroidogênese e, segundo Williams et al. (1989), pode influenciar o corpo lúteo a secretar progesterona, hormônio importante na preparação do ambiente uterino para a gestação (Hadley, 1996). 
Tabela 2. Médias e desvios-padrão de colesterol (mg/dl) de vacas Nelore doadoras de embrião, com escore corporal alto (7 a 9, em escala de 1 a 9), durante a superovulação com diferentes protocolos, Funilândia, 2003

\begin{tabular}{ccccc}
\hline \multirow{2}{*}{ Grupo } & \multicolumn{4}{c}{ Amostra } \\
\cline { 2 - 5 } & $\mathrm{A}$ & $\mathrm{B}$ & $\mathrm{C}$ & $\mathrm{D}$ \\
\hline $500 \mathrm{UI}$ de $\mathrm{FSH}^{1}$ & $131,22 \pm 30,15 \mathrm{aB}$ & $134,34 \pm 31,32 \mathrm{aA}$ & $163,64 \pm 60,95 \mathrm{aA}$ & $163,49 \pm 81,46 \mathrm{aA}$ \\
200mg de $\mathrm{FSH}^{2}$ & $103,01 \pm 21,83 \mathrm{aB}$ & $113,70 \pm 20,70 \mathrm{aA}$ & $110,32 \pm 26,62 \mathrm{aB}$ & $126,33 \pm 49,85 \mathrm{aA}$ \\
$180 \mathrm{mg} \mathrm{de} \mathrm{FSH}^{2}$ & $194,58 \pm 54,75 \mathrm{aA}$ & $148,79 \pm 53,11 \mathrm{aA}$ & $112,89 \pm 32,77 \mathrm{aAB}$ & $163,13 \pm 76,82 \mathrm{aA}$ \\
\hline
\end{tabular}

Letras diferentes, minúsculas na linha e maiúsculas na coluna, diferem entre si pelo teste Duncan $(\mathrm{P}<0,05)$. $\mathrm{CV}=$ $37,78 \%$. A = Antes do início da superovulação; B = Terceiro dia de superovulação - antes da aplicação de PGF2 $\alpha ; C$ $=$ Primeira inseminação artificial; $\mathrm{D}=$ Coleta de embriões.

${ }^{1}$ Pluset ${ }^{\circledR}$ - Laboratório Calier

${ }^{2}$ Foltropin-V® - Laboratório Vetrepharm

O valor médio encontrado para triglicérides, obtido da totalidade dos animais e das amostras coletadas, foi de $48,38 \pm 14,69 \mathrm{mg} / \mathrm{dl}$, valor acima do considerado normal para bovinos leiteiros durante o pré e o pós-parto (Marcos et al., 1990; Castillo, 1994; Zambrano, 2003) e próximo ao limite máximo sugerido por Rosemberger (1979). Os valores médios obtidos nos diferentes grupos reduziram, embora não apresentando significância, de antes do início da superovulação até a primeira inseminação artificial, com subseqüente estabilização até o momento da coleta dos embriões. Quando considerada a totalidade dos animais, as concentrações de triglicérides foram semelhantes às encontradas nos diferentes grupos, porém, apresentando significância, possivelmente por contemplar um número de animais $(\mathrm{n}=24)$ maior que cada grupo tratado com 500UI de FSH $(n=11), 200 \mathrm{mg}(\mathrm{n}=8)$ e $180 \mathrm{mg}$ de FSH $(\mathrm{n}=5)$. Não houve diferença entre os grupos $(\mathrm{P}>0,05)$ dentro de uma mesma amostra, para as concentrações de triglicérides, o que sinaliza não haver efeito do protocolo de superovulação utilizado sobre a concentração sangüínea desse parâmetro (Tab. 3).

Tabela 3. Médias e desvios-padrão de triglicérides $(\mathrm{mg} / \mathrm{dl})$ de vacas Nelore doadoras de embrião, com escore corporal alto ( 7 a 9, em escala de 1 a 9), durante a superovulação com diferentes protocolos

\begin{tabular}{lcccc}
\hline \multirow{2}{*}{ Grupo } & \multicolumn{4}{c}{ Amostra } \\
\cline { 2 - 5 } & $\mathrm{A}$ & $\mathrm{B}$ & $\mathrm{C}$ & $\mathrm{D}$ \\
\hline $500 \mathrm{UI}$ de $\mathrm{FSH}^{1}$ & $58,57 \pm 21,80 \mathrm{aA}$ & $51,08 \pm 12,16 \mathrm{aA}$ & $48,56 \pm 13,65 \mathrm{aA}$ & $48,17 \pm 16,00 \mathrm{aA}$ \\
$200 \mathrm{mg} \mathrm{de} \mathrm{FSH}^{2}$ & $50,96 \pm 13,85 \mathrm{aA}$ & $46,63 \pm 8,23 \mathrm{aA}$ & $42,58 \pm 10,87 \mathrm{aA}$ & $46,45 \pm 12,03 \mathrm{aA}$ \\
$180 \mathrm{mg} \mathrm{de} \mathrm{FSH}$ & $54,72 \pm 14,34 \mathrm{aA}$ & $51,17 \pm 11,66 \mathrm{aA}$ & $43,62 \pm 10,95 \mathrm{aA}$ & $41,46 \pm 13,02 \mathrm{aA}$ \\
\hline
\end{tabular}

Letras iguais, minúsculas na linha e maiúsculas na coluna, não diferem entre si pelo teste Duncan $(\mathrm{P}>0,05)$. $\mathrm{CV}=28,72 \%$. $\mathrm{A}=$ Antes do início da superovulação; $\mathrm{B}=$ Terceiro dia de superovulação - antes da aplicação de PGF2 $\alpha ; C=$ Primeira inseminação artificial; $\mathrm{D}=$ Coleta de embriões.

${ }^{1}$ Pluset ${ }^{\circledR}$ - Laboratório Calier

${ }^{2}$ Foltropin-V® - Laboratório Vetrepharm

A concentração sérica de triglicérides, superior à encontrada na literatura para bovinos de leite durante o pré e o pós-parto, aparentemente devese ao fato de os animais estarem apresentando balanço energético negativo, por estarem sendo alimentados durante o período de seca apenas com pastagem de Brachiaria brizanta e, apesar do escore corporal alto, podem ter equilibrado seu aporte energético pela mobilização de reservas corporais, primariamente do tecido adiposo, gerando um acúmulo de triglicérides na corrente circulatória (Zambrano, 2003). Segundo
Butler (2000), esses eventos podem estar associados com a redução da fertilidade pela diminuição da freqüência dos picos de LH (Canfield e Butler, 1990), presença de pequenos folículos dominantes (Murphy et al, 1991), ciclo estral com mais de três ondas foliculares e redução na produção de progesterona (Mann et al., 1996), características que não foram avaliadas neste experimento, por se tratar de um programa comercial no qual não foi permitido fazer modificações nos protocolos de rotina. 
Wathes et al. (1998) afirmaram que o aumento da concentração sérica de ácidos graxos está associado à elevação da concentração plasmática de colesterol. O número e o tamanho dos folículos, segundo Lucy et al. (1992), podem ser aumentados em animais que apresentam concentração de colesterol sangüíneo mais alta, sugerindo que o aumento da concentração de ácidos graxos pode ter efeito positivo na reprodução. Não está claro, com base na literatura consultada, se a elevação verificada na concentração de triglicérides nas doadoras poderia estar favorecendo ou não a resposta superovulatória.

A redução da concentração de triglicérides, antes do início da superovulação até a inseminação artificial, contrapõe-se ao encontrado por Vásquez-Añon (1996), que cita estar o processo de lipólise regulado pela concentração de glucocorticóides, catecolaminas, hormônio do crescimento, 17ß-estradiol e insulina. Assim, para animais superovulados, que apresentam concentração elevada de 17ß-estradiol no dia do estro (Sugano et al., 2001), não foi encontrada uma explicação plausível para a diminuição dos triglicérides por ocasião do estro, pois durante, o mesmo há expectativa de menor ingestão de alimento e, portanto, possibilidade de aumento da lipólise com conseqüente elevação da concentração de ácidos graxos.
Os valores encontrados para a concentração de progesterona no início da superovulação, no terceiro dia da superovulação e na inseminação artificial encontram-se dentro dos mencionados na literatura consultada, para vacas superovuladas, durante o ciclo estral e no pré e pós-parto (Borges, 2001; Mondal e Prakash, 2003). A concentração de progesterona por ocasião da coleta dos embriões foi inferior à encontrada por Sugano et al. (2001), possivelmente devido à baixa resposta das doadoras à superovulação, principalmente nos animais pertencentes ao grupo tratado com $200 \mathrm{mg}$ de FSH. A concentração de progesterona teve um pequeno aumento, antes do início da superovulação até o terceiro dia da mesma, devido ao desenvolvimento e à maturação do corpo lúteo (Mapletoft et al., 2000). A redução encontrada para progesterona, do terceiro dia da superovulação até a inseminação artificial, decorre da ação do análogo de PGF $2 \alpha$ utilizado, que causa luteólise e, com isso, possibilita a manifestação clínica de estro pelo animal (Milvae et al., 1996). O aumento encontrado para a progesterona, do momento da inseminação até a coleta dos embriões, pode ser atribuído à múltipla ovulação, em decorrência da administração do FSH, com formação de maior número de corpos lúteos e, com isso, aumento da secreção de progesterona (Tab. 4).

Tabela 4. Médias e desvios-padrão de progesterona $(\mathrm{mg} / \mathrm{dl})$ de vacas Nelore doadoras de embrião, com escore corporal alto ( 7 a 9, em escala de 1 a 9), durante a superovulação com diferentes protocolos

\begin{tabular}{lcccc}
\hline \multirow{2}{*}{ Grupo } & \multicolumn{4}{c}{ Amostra } \\
\cline { 2 - 5 } & $\mathrm{A}$ & $\mathrm{B}$ & $\mathrm{C}$ & $\mathrm{D}$ \\
\hline $500 \mathrm{UI}$ de $\mathrm{FSH}^{1}$ & $3,75 \pm 0,96 \mathrm{bA}$ & $4,13 \pm 1,12 \mathrm{bA}$ & $0,22 \pm 0,21 \mathrm{cA}$ & $6,59 \pm 5,13 \mathrm{aA}$ \\
$200 \mathrm{mg} \mathrm{de} \mathrm{FSH}^{2}$ & $3,59 \pm 1,05 \mathrm{abA}$ & $3,42 \pm 0,85 \mathrm{abA}$ & $0,32 \pm 0,46 \mathrm{bA}$ & $4,48 \pm 6,60 \mathrm{aA}$ \\
$180 \mathrm{mg}$ de FSH & $2,88 \pm 0,64 \mathrm{aA}$ & $3,59 \pm 1,08 \mathrm{aA}$ & $0,39 \pm 0,51 \mathrm{aA}$ & $3,42 \pm 4,89 \mathrm{aA}$ \\
\hline
\end{tabular}

Letras iguais, minúsculas na linha e maiúsculas na coluna, não diferem entre si pelo teste Duncan $(\mathrm{P}>0,05)$. $\mathrm{CV}=103,74 \%$. $\mathrm{A}=$ Antes do início da superovulação; $\mathrm{B}=$ Terceiro dia de superovulação - antes da aplicação de PGF $2 \alpha$; $=$ Primeira inseminação artificial; $D=$ Coleta de embriões.

${ }^{1}$ Pluset ${ }^{\circledR}$ - Laboratório Calier

${ }^{2}$ Foltropin-V® - Laboratório Vetrepharm

A elevação da concentração de progesterona após a ovulação é importante para o desenvolvimento embrionário, e a demora no aumento da secreção de progesterona está associada à redução da fertilidade (Mann et al., 1996). Animais que apresentam maior concentração de progesterona antes e depois da ovulação possuem maior taxa de gestação (Butler et al., 1996). Ashworth et al. (1989) sugeriram efeito da progesterona na modificação da maturação folicular e na qualidade do oócito, enquanto Lozano et al. (1998) consideraram também seu efeito sobre o ambiente uterino. Essas informações aparentemente não estão associadas à resposta superovulatória obtida neste experimento, pois foi verificada elevação 
da concentração de progesterona por ocasião da coleta dos embriões, principalmente nos animais que apresentaram boa resposta à superovulação.

Os valores encontrados para a concentração de aspartato transaminase (AST), do início da superovulação até a coleta dos embriões, foram inferiores aos encontrados por Doornenbal et al. (1988) para bovinos de corte da raça Brahman, mas dentro dos limites estabelecidos por Rosenberger (1979) (Tab. 5).

Tabela 5. Médias e desvios-padrão de aspartato transaminase $(\mu \mathrm{n} / \mathrm{ml})$ de vacas Nelore doadoras de embrião, com escore corporal alto (7 a 9, em escala de 1 a 9), durante a superovulação com diferentes protocolos

\begin{tabular}{|c|c|c|c|c|}
\hline \multirow{2}{*}{ Grupo } & \multicolumn{4}{|c|}{ Amostra } \\
\hline & A & B & $\mathrm{C}$ & $\mathrm{D}$ \\
\hline 500UI de $\mathrm{FSH}^{1}$ & $67,45 \pm 27,47 \mathrm{aA}$ & $63,73 \pm 12,85 \mathrm{aA}$ & $65,18 \pm 14,85 \mathrm{aA}$ & $73,40 \pm 19,87 \mathrm{aA}$ \\
\hline $200 \mathrm{mg}$ de $\mathrm{FSH}^{2}$ & $65,25 \pm 16,87 \mathrm{aA}$ & $62,25 \pm 18,81 \mathrm{aA}$ & $64,00 \pm 18,88 \mathrm{aA}$ & $63,86 \pm 14,95 \mathrm{aA}$ \\
\hline $180 \mathrm{mg}$ de $\mathrm{FSH}^{2}$ & $61,00 \pm 33,95 \mathrm{aA}$ & $76,80 \pm 50,69 \mathrm{aA}$ & $78,60 \pm 30,26 \mathrm{aA}$ & $75,00 \pm 32,02 \mathrm{aA}$ \\
\hline
\end{tabular}

Letras iguais, minúsculas na linha e maiúsculas na coluna, não diferem entre si pelo teste Duncan $(\mathrm{P}>0,05)$. $\mathrm{CV}=33,90 \%$. $\mathrm{A}=$ Antes do início da superovulação; $\mathrm{B}=$ Terceiro dia de superovulação - antes da aplicação de PGF2 $\alpha$; $=$ Primeira inseminação artificial; $\mathrm{D}=$ Coleta de embriões.

${ }^{1}$ Pluset-V® - Laboratório Calier

${ }^{2}$ Foltropin ${ }^{\circledR}$ - Laboratório Vetrepharm

A não-alteração da concentração de AST, de antes do início da superovulação até a coleta dos embriões e entre os tratamentos com diferentes protocolos, indica que a superovulação não interferiu na atividade hepática, não sendo esta, aparentemente, a causa da variação na concentração de triglicérides por modificação em sua via metabólica (Lehninger et al., 1995).

\section{CONCLUSÃO}

O tratamento com 500UI de FSH e 200 e $180 \mathrm{mg}$ de FSH não alterou o metabolismo de vacas Nelore doadoras de embrião e com escore corporal alto, refletido na concentração de colesterol, triglicérides, AST e progesterona, do início da superovulação até a coleta dos embriões.

\section{AGRADECIMENTOS}

Os autores agradecem a Cauêmbrio pela oportunidade de realização deste trabalho, a Quibasa pela doação de kits comerciais para análises bioquímicas e ao laboratório Betlab pela realização das análises de progesterona.

\section{REFERÊNCIAS BIBLIOGRÁFICAS}

ASHWORTH, C.J.; SALES, D.I.; WILMUT, I. Evidence of an association between the survival of embryos and the periovulatory plasma progesterone concentration in the ewe. J. Reprod. Fert., v.87, p.2332, 1989.

BORGES, A.M. Influência de diferentes manejos $e$ tratamentos hormonais na dinâmica ovariana durante o ciclo estral e no anestro pós-parto de vacas Gir e Nelore. 2001. 145f. Tese (Doutorado) - Universidade Federal de Viçosa, Viçosa.

BORGES, A.M.; TORRES, C.A.A.; RUAS, J.R.M. et al. Concentração plasmáticas de colesterol total e lipoproteína de alta densidade em novilhas mestiças doadoras de embriões tratados com somatotropina recombinante. Arq. Bras. Med. Vet. Zootec., v.53, p.605-610, 2001.

BUTLER, W.R.; CALLAMAN, J.J.; BEAM, S.W. Plasma and milk urea nitrogen in relation to pregnancy rate in lactating dairy cattle. J. Anim. Sci., v.74, p.858865, 1996.

BUTLER, W.R. Nutritional interactions with reproductive performance in dairy cattle. Anim. Reprod. Sci., v.60-61, p.449-457, 2000.

CANFIELD, R.W.; BUTLER, W.R. Energy balance and pulsatile LH secretion in early postpartum dairy cattle. Domest. Anim. Endocrinol., v.7, p.323-330, 1990.

CASTILlO, O. A. C. Aspectos bioquímicos do sangue e do leite de vacas leiteiras no período seco e no início da lactação. 1994. 77f. Dissertação (Mestrado) Escola de Veterinária, Universidade Federal de Minas Gerais, Belo Horizonte.

DIAS, F.M.G.N. Efeito da condição corporal razão pesolaltura e peso vivo sobre o desenvolvimento reprodutivo pós-parto de vacas de corte zebuinas, 
1991, 100f, Dissertação (Mestrado) - Escola de Veterinária, Universidade Federal de Minas Gerais, Belo Horizonte.

DOORNENBAL, H.; TONG, A.K.W.; MURRAY, N.L. Reference values of blood parameters in beef cattle of different ages an stages of lactation. Can. J. Vet. Res., v.52, p.99-105, 1988.

GUÉDON, L.; SAUMANDE, J.; DUPRON, F. et al. Serum cholesterol and triglycerides in postpartum beef cows and their relationship to the resumption of ovulation. Theriogenology, v.51, p.1405-1415, 1999.

HADLEY, M.E. Endocrinology. 4 ed., Tucson: Prentice Hall, 1996. 518p

LEHNINGER, A.L.; NELSON, L.D.; COX, M.M. Princípios de Bioquímica. 8 ed., São Paulo: Sarvier, 1995. 839p.

LOZANO, J.M.; ABECIA, J.A.; FORCADA, F. et al. Effect of undernutrition on the distribution of progesterone in the uterus of ewes during yhe luteal phase of the estrous cycle. Theriogenology, v.49, p.539-546, 1998 .

LUCY, M.C.; SAVIO, J.D.; De La SOTA, R.L. et al. Factors that affect ovarian follicular dynamics in cattle. J. Anim. Sci.,v.70, p.3615-3626, 1992.

MANN, G.E.; MANN, S.J.; LAMMING, G.E. The inter-relationship between maternal hormone environment and the embryo during early stages of pregnancy. J. Reprod. Fert., v.21, p.37, 1996.

MAPLETOFT, R.J.; BÓ, G.A.; ADAMS, G.P. Avanços na manipulação do ciclo estral de doadoras e receptoras nos programas de TE em bovinos. Arq. Fac. Vet. UFRS, v.28, supl., p.24-51, 2000.

MARCOS, E.; MAZUR, A.; CARDOT, P. The effect of pregnancy and lactation on serum lipid and apolipoprotein B and A-I levels in dairy cows. $J$. Anim. Physiol. Anim. Nutr., v.64, p.133-138, 1990.

MILVAE, R. A.; HINCKLEY, S. T.; CARLSON, J. C. Luteotropic and luteolytic mechanisms in the bovine corpus luteum. Theriogenology, v.45, p. 13271349, 1996

MONDAL, S; PRAKASH, B.S. Peripheral plasma progesterone concentration in relation to estrus expression in Sahiwal cows. Indian J. Physiol. Pharmacol., v.47, p.111-114, 2003.

MURPHY, M.G.; ENRIGHT, W.J.; CROWE, M.A. et al. Effect of dietary intake on pattern of growth of dominant follicles during the oestrous cycle in beef heifers. J. Reprod. Fert., v.92, p,333-338, 1991.

NEVES, E.F.; RAMOS, A.F.; MARQUES JUNIOR, A.P. Pré-tratamento com somatotropina bovina (rbST) na superovulação de doadoras da raça Holandesa. Arq. Bras. Med. Vet. Zootec., v.57, p.205-209, 2005.

ROSENBERGER, G. Clinical examination of cattle. Verlag Paul Parey, Berlin: W. B. Saunders Company, Philadelphia. 1979. 453p.

RUAS, J.R.M. Eficiência reprodutiva e perfil metabólico de vaca zebu em relação a status reprodutivo, condição corporal, amamentação diferenciada e suplementação alimentar. 1998. $105 \mathrm{f}$. Tese (Doutorado) - Universidade Federal de Viçosa, Viçosa.

SISTEMA de análises estatísticas e genéticas - SAEG. Viçosa: UFV, 1995.

SUGANO, M.; SHINOGI, T.; NAKADA, K. et al. Endocrine profiles and embryo quality in Japanese Black cattle superovulated with human menopausal gonadotropin and porcine follicle stimulating hormone. Reprod. Dom. Anim., v.36, p.57-63, 2001.

VÁZQUEZ-AÑON, M. Adipose tissue metabolism in periparturient cows: relationship to the development of fatty liver. 1996. 170f. Thesis (PhD) - University of Wisconsin-Madison, WI.

WATHES, D.C.; ROBINSON, R.S.; PUSHPAKUMARA, A. et al. Nutritional effects on reproductive performance in dairy cows. Cattle Pract., v.6, p.371-377, 1998.

WILLIAMS, G.L. Modulation of luteal activity in postpartum beef cows through changes in dietary lipid. J. Anim. Sci., v.67, p.785-793, 1989.

ZAMBRANO, W.J. Perfil metabólico de novilhas mestiças leiteiras no pré-parto e até o quinto mês de lactação. 2003. 147f. Tese (Doutorado) - Escola de Veterinária, Universidade Federal de Minas Gerais, Belo Horizonte. 